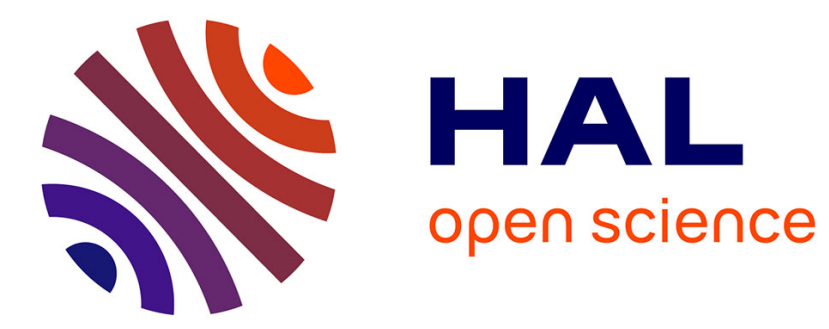

\title{
L'OMC, au-delà des fantasmes
}

Patrick Messerlin

\section{To cite this version:}

Patrick Messerlin. L'OMC, au-delà des fantasmes. Critique Internationale, 2000, 6, pp.38-46. 10.3406/criti.2000.1372 . hal-01011208

\section{HAL Id: hal-01011208 \\ https: / hal-sciencespo.archives-ouvertes.fr/hal-01011208}

Submitted on 23 Jun 2014

HAL is a multi-disciplinary open access archive for the deposit and dissemination of scientific research documents, whether they are published or not. The documents may come from teaching and research institutions in France or abroad, or from public or private research centers.
L'archive ouverte pluridisciplinaire HAL, est destinée au dépôt et à la diffusion de documents scientifiques de niveau recherche, publiés ou non, émanant des établissements d'enseignement et de recherche français ou étrangers, des laboratoires publics ou privés.

\section{(이)(\$)}

Distributed under a Creative Commons Attribution - NonCommercial - NoDerivatives| 4.0 


\section{L'OMC, au-delà des fantasmes}

par Patrick A. Messerlin

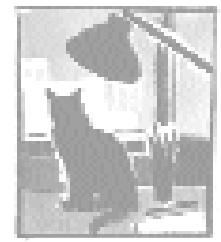

Contre-jour

l

Conférence ministérielle (la « Ministérielle ») de l'Organisation mondiale du commerce de Seattle (décembre 1999) a révélé en France, dès sa préparation, des lignes de fracture qui se retrouvent, à bien des égards, aux États-Unis. Face à un large consensus des partis de « gouvernement », des dirigeants politiques (Pat Buchanan et Charles Pasqua) font de l'opposition à l'OMC leur principal cheval de bataille - avec des retombées « antifédérales » contre Washington ou contre Bruxelles. À côté des politiques, des organisations non gouvernementales $(\mathrm{ONG})$ utilisent Seattle pour afficher leurs préoccupations - prenant généralement à partie l'OMC mais aussi, parfois, apportant leur soutien à certains de ses résultats, tels que la suppression des subventions agricoles à l'exportation décidée lors de l'Uruguay Round et saluée par la Confédération paysanne.

La population française demeure, jusqu’à présent, « consentante » à la progression de la mondialisation. Cette observation peut étonner, car elle va à l'encontre d'une France souvent perçue comme « protectionniste ». Pourtant, un sondage d'opinion peu remarqué faisait bien apparaître que plus de $40 \%$ des Français sont en faveur de l'ouverture des échanges. La question ne laissait guère de place à l'ambiguïté : « Laquelle des deux approches suivantes estimez-vous la meilleure pour améliorer la situation économique et celle de l'emploi dans notre pays : protéger notre industrie en restreignant les importations, ou éliminer les restrictions à l'importation pour accroître notre commerce international ? >1 . Cet instantané ne manque pas d'humour car il montre une France très anglo-saxonne : la proportion de réponses favorables à l'ouverture des échanges est à peu près la même chez les Britanniques et les Américains.

Que le débat suscité par la Ministérielle de Seattle fasse apparaître une France plus ouverte qu'on ne le croit souvent est sain. Reste que cette réunion amorce le Round le plus difficile qui ait été lancé depuis la Seconde Guerre mondiale, et cela pour trois raisons : les négociateurs devront désormais travailler sous l'œil des médias ; ils devront aborder les problèmes de protection laissés de côté par les Rounds précédents, car particulièrement épineux ; et ils feront face à de nouveaux sujets que les États membres eux-mêmes ont du mal à gérer, alors même que l'OMC est accusée de limiter la souveraineté de ces États. 


\section{Travailler sous l'œil des médias et des ONG}

La Ministérielle de Seattle est la première conférence de l'OMC ayant pour objet de fixer un « agenda » (ordre du jour) sous les feux de la rampe. Presque tout le monde a oublié la Ministérielle de 1982 qui devait lancer un nouveau cycle de négociations (devenu par la suite l'Uruguay Round) et qui se termina de façon calamiteuse, puisque certains pays membres de ce qui était alors le GATT, dont l'Australie, quittèrent la réunion. Il fallut quatre ans et un groupe de travail remarquable (le rapport Leutwiller) pour réparer les dégâts et relancer le processus à la conférence de Punta del Este de 1986. Mais, à cette époque, on travaillait avec une couverture médiatique modeste, sur des sujets bien délimités, comme les barrières aux frontières (droits de douane et autres restrictions aux échanges), et exclusivement entre négociateurs rompus à ces exercices.

La Ministérielle de Seattle est un « animal » totalement différent : pas tant par le nombre de pays (135, deux fois plus qu'en 1982) car ceux-ci ont, de nos jours, des visions de la mondialisation plus proches les unes des autres qu'en 1982, que par l'importance de sa « couverture » (l'événement apparaissant régulièrement dans la presse écrite et audiovisuelle pendant tout le mois précédant la réunion) et par l'irruption, sur le devant de la scène médiatique, de groupes de pression d'un type nouveau, avec plus de $700 \mathrm{ONG}$ « accréditées » auprès de la réunion. Ces ONG, qui se caractérisent par la défense d'intérêts précis, parfois mal pris en compte par les États (comme l'environnement ou la sécurité alimentaire), se sont multipliées depuis une dizaine d'années, portées par le progrès technique des télécommunications. On les qualifie souvent de représentatives de la «société civile », ce qui est excessif car, après tout, aucune d'entre elles n'est issue d'un vote démocratique ni responsable devant des électeurs. En fait, nombre d'entre elles ne sont guère autre chose que des lobbies traditionnels - fonction au demeurant fort utile - comme le commerce international en connaît des millions, et sont d'ailleurs coalisées avec de tels lobbies.

Or les négociateurs - notamment ceux de la « Quad», qui regroupe le Canada, la Communauté européenne (CE), les États-Unis et le Japon - n'ont pris conscience des contraintes imposées par cette couverture médiatique que tardivement et, sans doute, incomplètement.

Ce décalage est apparu clairement avec l'annonce, au printemps 1999, par les pays de la Quad, de l'année 2003 comme date souhaitable de la fin des négociations à venir. Ce message s'adressait surtout aux négociateurs eux-mêmes, hantés par le souvenir des huit ans de l'Uruguay Round (en fait six années vraiment actives, le processus ayant été en sommeil en 1991 et 1992). Les discussions, qui se déroulent toujours à Genève, soumettent les participants à deux formes d'épreuves. Les représentants des pays les plus riches ou les plus intéressés au commerce 
international doivent faire des allers et retours épuisants entre Genève (pour négocier) et leur capitale (pour informer les administrations centrales et les industriels concernés, et participer à l'élaboration de la position de leur pays). Par contre, les négociateurs des pays pauvres ou moins directement concernés sont des diplomates en poste à Genève (parfois même ailleurs), paralysés par l'absence d'instructions ou même d'informations sur ce que souhaite leur gouvernement. Les uns et les autres redoutent de longues négociations.

Mais cette annonce d'une date-limite a eu deux conséquences négatives. D'une part, elle ne tenait pas compte du calendrier des pays autres que ceux de la Quad, notamment de celui des pays en voie de développement. Or l'un des principaux résultats de l'Uruguay Round pour ces derniers est l'élimination des barrières non tarifaires (Accord multifibres) imposées par les pays industrialisés sur les importations d'habillement, élimination qui ne sera acquise qu'en 2005. Un autre résultat important est la fin des subventions agricoles à l'exportation, pour laquelle l'année 2003 est une première étape. Pour ces deux raisons, il paraît difficile d'imaginer que de grands exportateurs, effectifs ou potentiels, d'habillement, comme la Chine (quand elle sera membre de l'OMC) ou l'Inde, puissent accepter de signer un nouveau Round avant que l'ancien ne soit complètement mis en œuvre. Et l'on voit mal, aussi, les nombreux pays émergents ou en développement ayant des intérêts agricoles, soit à l'exportation, soit à la reconstruction de leur agriculture dévastée par les subventions agricoles des pays riches, ne pas attendre un ou deux ans après 2003 pour s'assurer d'une mise en œuvre robuste des accords de l'Uruguay Round.

En même temps qu'elle irritait les pays en développement, l'annonce de la datelimite de 2003 a exacerbé les ardeurs des ONG, convaincues que le temps leur était compté et qu'elles devaient agir vite et fort pour se faire entendre. Par un effet de domino, les opinions publiques ont eu l'impression que ce nouveau Round serait appliqué dès sa signature, impression exploitée par les mouvements souverainistes dans les pays où ils existent. Peu de gouvernements ont rappelé que non seulement l'aboutissement des négociations n'est pas un processus linéaire (tant s'en faut), mais aussi que la mise en œuvre de leurs résultats prend beaucoup de temps. Ainsi, l'Uruguay Round a été commencé en 1982, véritablement lancé en 1986, au bord du désastre en 1990 (avec l'échec de la Conférence de Bruxelles) et achevé en 1994 ; et son application s'étale de 1995 à 2001 pour le plus aisé, mais se prolongera jusqu'en 2005 pour les points difficiles : vingt-trois ans en tout!

Une Ministérielle qui, comme celle de Seattle, lance un nouveau Round sous l'œil des médias doit affronter un dernier obstacle. Par définition, chaque pays hausse le ton au moment de l'établissement d'un agenda de négociations. Ce faisant, il montre le « prix » que ses partenaires devront payer pour avoir un accès plus large à ses marchés : tout le monde sait bien que chacun va devoir en rabattre, mais il ne faut pas le dire trop vite. La présence des médias, si elle offre un excellent 
instrument pour afficher son « prix initial », est en même temps un piège collectif, chacun s'arc-boutant sur son prix par crainte de donner l'impression de céder trop vite. Un piège de ce type avait déjà englouti la Ministérielle de 1982.

Toutefois, l'expérience de l'Uruguay Round a montré que le véritable agenda se fait beaucoup plus au fur et à mesure des négociations que lors de la Ministérielle de départ. Cela découle d'abord des lois de toute négociation entre États souverains : pour qu'un Round soit accepté et ratifié, chaque pays doit y trouver un intérêt et, si les pourparlers durent des années, ce qui intéresse un pays à la fin peut être fort différent de ce qu'il cherchait à obtenir au début. Mais cela découle aussi, et sans doute surtout, des tractations internes qui accompagnent, au sein de chaque pays, la négociation internationale - c'est-à-dire des coalitions internes qui, à un moment donné, sont prêtes à soutenir un gouvernement donné dans son entreprise d'ouverture économique. Par exemple, en 1986, le gouvernement américain s'appuyait sur des intérêts favorables à la libéralisation des investissements internationaux et faisait de ce point une priorité ; huit ans plus tard, cette coalition s'était effondrée, et le gouvernement concentrait ses efforts sur les services et les droits de propriété intellectuelle, deux sujets soutenus par des coalitions différentes de celle qui appuyait l'investissement international.

\section{Travailler sous contrainte de résultats}

La Ministérielle de Seattle présente une autre nouveauté : elle est la première dont une partie de l'agenda (l'« agenda intégré » ou built-in agenda) a été fixée par le précédent Round. Il a en effet été convenu en 1994 que l'agriculture et les services feraient l'objet de nouvelles négociations, à commencer en 2000 ; et que, à ces deux sujets centraux (représentant une part du PIB double de celle de l'industrie qui a, seule, fait l'objet de négociations de libéralisation entre 1948 et 1986), devrait s'ajouter un examen de l'état des lieux dans d'autres domaines (droits de propriété intellectuelle, accord sur l'investissement, examen des politiques commerciales), créant autant d'incitations à négocier.

Cette pré-fixation de l'agenda peut paraître étrange. L'explication en semblera encore plus étrange à ceux qui ne suivent que de loin le commerce international : tout compte fait, l'Uruguay Round a peu libéralisé. Il a surtout fait œuvre « constitutionnelle », en posant les règles de droit qui doivent présider aux libéralisations futures. Par exemple, l'accord agricole qui en est issu n'impose en réalité que de transformer la protection opaque d'avant 1995 en une protection plus transparente. Ainsi, le niveau de protection (« coefficient nominal de soutien aux producteurs ») de la Communauté européenne pour les grands produits agricoles (céréales, lait, viande, sucre), estimé par l'OCDE à $86 \%$ en 1986-1988, est encore de $83 \%$ en 1998, et celui des États-Unis est passé de 35 à 28 \% sur la même période. En ce 
qui concerne l'industrie (objet des libéralisations passées), le chiffre moyen de 5 à $6 \%$ pour les droits de douane européens ou américains est une information si partielle qu'elle donne une vue fausse de la réalité. D'une part, elle ignore les nombreux instruments de protection autres que les droits de douane, d'autre part, il s'agit d'une moyenne pondérée par les importations : ce qui sous-estime systématiquement le niveau réel de protection, puisqu'on pondère des barrières élevées par des importations sous contrainte de ces mêmes barrières, donc réduites voire nulles, et des barrières faibles par des importations « normales », voire amplifiées pour cause de substitution.

Une estimation tenant compte de tous ces aspects pour l'agriculture et l'industrie donne un niveau de protection globale de l'ordre de 13 à $14 \%$ pour la Communauté (10 à $12 \%$ pour les États-Unis, moins protégés en agriculture). Non seulement ce niveau est donc bien plus élevé qu'on ne le dit, mais il est aussi relativement stable depuis 1990.

Enfin, l'Accord général sur les services (GATS) se limite à un accord-cadre constitutionnel qui ne s'est accompagné d'aucune libéralisation effective de quelque ampleur (même dans les services financiers et de télécommunications).

Ces observations ont deux corollaires. D'une part, elles soulignent l'ampleur de la tâche du prochain Round. Comme le niveau de protection varie énormément par secteur (avec des pointes pouvant aller jusqu'à $100 \%$ comme dans les céréales, les viandes, le tabac ou certaines industries agro-alimentaires) et que la protection existante crée d'énormes rentes souvent captées par les producteurs protégés, les négociateurs devront s'attaquer à des « forteresses de protection » qui ont su résister à des décennies de libéralisation et qui ont les moyens financiers d'influencer leurs gouvernements. D'autre part, ces observations soulignent l'intérêt économique d'un nouveau Round : une telle protection coûte cher, en gros 5 \% du PIB (soit, pour l'Europe, presque le PIB espagnol).

\section{Ouvrir de nouveaux chantiers}

Toutes ces observations sur la persistance de la protection et la lenteur de son érosion amènent à se demander ce qui peut bien alors nourrir l'impression, si répandue, d'une forte libéralisation au cours des quinze dernières années.

Une première réponse tient aux investissements directs internationaux, en très forte expansion depuis le milieu des années quatre-vingt : le taux annuel de croissance des opérations de fusion-acquisition est en gros de $25 \%$ entre 1986 et 1997, contre $10 \%$ pour le commerce des biens et services. À travers ces investissements, les multinationales, qu'elles soient étrangères ou françaises, apportent une vision nouvelle des marchés sur lesquels elles pénètrent. Elles ont des idées et des audaces que les firmes locales n'ont pas eues pendant des années, et, en ce sens, elles sym- 
bolisent une « présence » internationale plus forte dans le pays en question que de simples échanges de biens.

Cette présence peut être perçue comme une atteinte à la souveraineté. Mais une telle réaction apparaît incroyablement fruste (et incohérente quand elle s'accompagne de fierté nationale à propos de la politique menée par Renault chez le producteur japonais Nissan). Car, en achetant une marque du pays dans lequel elle entre, une multinationale lui permet souvent de croître en l'intégrant dans sa stratégie mondiale. Qui défend alors la souveraineté ? L'entreprise «nationale » qui n'a pas su développer le produit, ou la multinationale « étrangère » qui le fait ? De plus, contrairement aux idées reçues, les multinationales ne cherchent pas au loin ce qu'elles peuvent trouver tout près. Le cas de MacDo est emblématique de cette approche rudimentaire : souvent pris à partie, c'est en fait une firme on ne peut plus « française » en France, puisqu'elle repose sur des entrepreneurs et travailleurs français, et à plus de $95 \%$ sur des produits alimentaires français.

Il existe une autre explication à cette impression de forte mondialisation : le progrès technique, notamment dans les télécommunications et les biotechnologies. C'est, sans nul doute, la source des peurs les plus fortes, d'où son exploitation politique intense.

L'ampleur du progrès technique que nous connaissons depuis une vingtaine d'années est difficilement mesurable, mais on peut s'en faire une idée dans le cas des télécommunications : le coût d'une communication téléphonique transatlantique a été presque divisé par cent entre 1945 et 1990 - alors que, dans le même laps de temps, la protection douanière n'a baissé tout au plus que d'un facteur dix. Les exemples allant dans le même sens sont légion. Ainsi l'utilisation d'Internet fait beaucoup plus pour renforcer la concurrence entre les librairies (aujourd'hui), les concessionnaires automobiles ou les cinémas ou chaînes de télévision (demain) que tout ce qui a pu (ou pourra) être fait en matière de libéralisation.

Le progrès technique comme force principale de la mondialisation suscite des peurs instinctives : c'est cela qui se prête à l'exploitation politique. Il est tout à fait frappant que les ONG soucieuses de l'environnement se soient vu ravir la vedette par les ONG centrées sur la sécurité alimentaire. Comment interpréter ce changement ? Il peut certes tenir à ce que des débuts de réponse ont été apportés aux problèmes environnementaux d'ordre mondial. Mais il tient davantage du sentiment de plus grande « proximité » du danger alimentaire dans l'opinion publique. Il tient encore - et surtout - aux liens entre les ONG « alimentaires » et les groupes de pression traditionnels des agriculteurs, et aux relations étroites entre ces derniers et les milieux politiques. Ces groupes d'intérêt sont beaucoup plus soucieux de dramatiser les situations et de désigner comme bouc émissaire une institution qui porte atteinte à leurs intérêts très privés de producteurs que d'œuvrer à un réel assainissement de la production alimentaire, comme en témoignent les 
déboires récents de l'agriculture biologique en France (comment produire « biologique » dans un environnement pollué ?).

Pour paradoxal que cela puisse paraître, l'OMC est l'institution la mieux à même de répondre aux problèmes de santé alimentaire, voire d'environnement.

En effet, la «mal-bouffe », pour rester en Europe où se situent la plupart des accusateurs, est la conséquence directe non de l'OMC, mais de l'agriculture intensive imposée par la Politique agricole commune (PAC). Depuis son origine, les économistes soulignent que la PAC favorise l'utilisation massive d'engrais ou de techniques d'élevage à risque, aux graves conséquences écologiques. L'accord agricole de l'Uruguay Round, en prévoyant la suppression des subventions à l'exportation et le maintien des seules subventions « découplées » (assurant un revenu minimum aux agriculteurs sans les inciter à accroître leur production) ont constitué, dès 1994, une réponse à la «mal-bouffe ».

Reste le problème récemment apparu des organismes génétiquement modifiés (OGM). Jusqu'ici, chaque État membre de l'OMC (donc l'OMC elle-même) applique un principe, celui de faire confiance aux scientifiques pour faire la différence entre des mesures de précaution « raisonnables » (acceptables aux yeux de l'OMC) et des mesures « déraisonnables », qui cherchent plus à protéger les producteurs agricoles que la sécurité alimentaire (que l'OMC refuse de prendre en considération). Seulement, ce « principe de confiance » dans les scientifiques pose problème en période de progrès technique intense. Dans des conflits impliquant des techniques bien connues, comme celui du bœuf aux hormones, l'OMC peut légitimement l'utiliser : il existe une opinion dominante chez les scientifiques, celle de l'innocuité de ces hormones - certaines, d'ailleurs, sont autorisées en Europe à titre curatif (!) alors même que le processus européen de surveillance de l'interdiction est pour le moins imparfait. Par contre, dans le cas des OGM, la communauté scientifique est divisée : le progrès est tellement récent et fulgurant qu'il est difficile de chiffrer, sous forme de probabilités, les risques à long terme que peuvent comporter ces techniques. En cas de conflit (qui ne s'est pas encore produit), il n'y a pas de gestion possible des risques, et l'OMC n'a plus de base légitime solide sur laquelle s'appuyer.

Cette incapacité ne lui est pas propre : tous les États membres en sont frappés. Certains d'entre eux avancent le « principe de précaution ». Mais celui-ci n'est pas un progrès par rapport au « principe de confiance » : il ne fait que déplacer la « majorité » requise chez les scientifiques consultés - d'une opinion dominante vers une opinion plus ou moins unanime - sans répondre au problème fondamental de l'impossibilité de calculer le risque. Il établit de plus un système de veto conduisant à la prohibition prolongée de nouvelles technologies, ce qui favorise des intérêts très privés, comme cela a été mentionné lors de l'affaire du sang contaminé, comme cela a été le cas dans l'affaire du bœuf aux hormones (l'interdiction de ces 
hormones a été adoptée pour stopper la croissance des stocks européens de viande, non pour des raisons sanitaires) et comme cela pourrait l'être pour les OGM. La crédibilité du principe de précaution s'en trouve fragilisée, particulièrement dans un pays qui ne s'en est guère embarrassé pour développer massivement l'énergie nucléaire.

Que peut apporter alors l'OMC ? La réponse tient dans sa philosophie : offrir le plus grand choix possible à chacun, en « labélisant » les produits étrangers et locaux de façon non discriminatoire. Chaque Européen pourra choisir entre du bœuf aux hormones et du bœuf sans hormones, et chaque Américain entre du fromage pasteurisé et du fromage non pasteurisé. À ces liberté et responsabilité individuelles en matière de choix fait écho une responsabilité publique, celle d'assurer une information honnête (fondée sur un jugement, et non sur une décision, de la communauté scientifique de chaque État membre) et d'assurer la traçabilité des produits étrangers et locaux.

\section{OMC et souveraineté}

On ne peut parler de la Ministérielle de Seattle sans évoquer la question de la perte de souveraineté qu'imposerait l'OMC. Sur le plan juridique, cette critique est un non-sens. L'OMC n'a d'autre pouvoir que celui que veulent bien lui donner ses membres, qui sont des États souverains. Sa seule règle est qu'un État doit être cohérent : il doit respecter les engagements qu'il a signés. L'Europe a été obligée de payer des compensations aux États-Unis pour les affaires de la banane et du bœuf aux hormones simplement parce que, dans ces deux affaires, l'Europe ne respectait pas ses propres engagements. En fait, l'OMC peut être assimilée à un contrat qu'un État a signé et dont d'ailleurs il peut se dégager, en payant des compensations.

Sur le plan politique, l'OMC ne fonctionne que sur le principe de la réciprocité : un État signe des engagements parce que, en échange, il obtient de ses partenaires des avantages qu'il estime préférables au statu quo. Il est d'ailleurs amusant d'observer le débat français sur la souveraineté à un moment où la Chine, un pays pourtant particulièrement chatouilleux en la matière, entre sans état d'âme à l'OMC - sans doute parce qu'elle a compris que le degré de liberté (de souveraineté) permis par cette institution est considérable : il n'a pour limite que la capacité du pays à trouver des solutions non discriminatoires aux problèmes posés. En somme, il n'a pour limite que l'imagination du pays, son intelligence.

Post-scriptum, après Seattle (7 décembre). Contrairement à la Ministérielle de 1982, celle de Seattle s'est terminée par une « suspension » des négociations, celles-ci devant reprendre en janvier 2000 : il est donc encore trop tôt pour parler d'un échec, 
d'autant que les textes élaborés à Seattle paraissaient proches d'un compromis acceptable par tous. Ce délai supplémentaire sera-t-il mis à profit ? On peut l'espérer car tout le monde a quitté Seattle avec des regrets : les Américains, furieux de l'insistance de leur Président sur les normes sociales ; les Européens, penauds de leur incapacité à cacher leurs dissensions internes ; le Groupe de Cairns et les Asiatiques, exaspérés de ne pas avoir été entendus sur des points essentiels pour eux ; les pays en développement, bien décidés à revenir mieux préparés ; et même les ONG, qui commencent à comprendre que leur survie dépend de celle de l'OMC (puisqu'elles veulent une place dans cette institution).

1. The Economist, 2 janvier 1999, p. 53.

\section{Bibliographie}

Jacquet (P.), Messerlin (P.A.) et Tubiana (L.), 1999. Le cycle du millénaire, Conseil d'analyse économique, Premier ministre, Paris, La Documentation française.

MesSERLIN (P.A.), à paraître. Measuring the Costs of Protection in Europe, Washington, Institute for International Economics.

ScHOTт (J.J.), à paraître. The World Trading System : Seattle and Beyond, Washington, Institute for International Economics. 